\title{
BARRERAS Y OPORTUNIDADES PARA LA ADOPCIÓN DE PRÁCTICAS DE MANEJO FORESTAL SOSTENIBLE EN LA AMAZONIA RESULTADOS DE UN ESTUDIO EN BRASIL, BOLIVIA Y PERÚ
}

\author{
César Sabogal ${ }^{1}$, Laura Snook², Marco Boscolo ${ }^{3}$, Benno Pokorny ${ }^{4}$, \\ Marco Lentini ${ }^{5}$, Lincoln Quevedo ${ }^{6}$ y Violeta Colán 7
}

\section{RESUMEN}

A pesar de las cuantiosas inversiones en investigación, asistencia técnica y capacitación, la adopción de prácticas de manejo forestal sostenible (MFS) en los trópicos permanece todavía en niveles muy bajos. En la Amazonia, se conoce mucho más de lo que actualmente es aplicado acerca del manejo de los bosques para la producción de madera. En el año 2001, se inició un proyecto de investigación para analizar el nivel de adopción de un conjunto de prácticas claves de MFS por parte de empresas madereras en Bolivia, Brasil y Perú. Dicho proyecto buscaba también determinar los factores que influyen en la adopción de esas prácticas. Con base en los resultados y lecciones obtenidas, el proyecto se propuso derivar estrategias para aumentar la adopción del buen manejo forestal en escala empresarial. Para este fin se empleó varias metodologías; una revisión del marco regulatorio para el manejo forestal en cada país; entrevistas a gerentes o propietarios de empresas y sus profesionales forestales (94 empresas en Brasil, 23 concesionarios en Bolivia, 66 productores en Perú), evaluaciones de campo sobre la aplicación de las prácticas seleccionadas (27 proyectos de manejo forestal en Brasil, 11 en Bolivia y 13 en Perú) y talleres con diversos actores del sector forestal.

Se encontró que en los tres paises, las tasas de adopción de las prácticas de manejo son muy variables. En Brasil, se obtuvo una tasa promedio de adopción de $56 \%, 57 \%$ para Bolivia y $41 \%$ para Perú, aunque algunas prácticas son adoptadas más ampliamente en Brasil que en Bolivia o Perú. Estas prácticas, relacionadas principalmente con la planificación, incluyen el respeto a las áreas de reserva o de protección permanente, el control de la caza, el censo comercial, la protección del área de manejo, la planificación de caminos, la protección de los árboles de futura cosecha, el corte de lianas pre-aprovechamiento y los tratamientos silviculturales para promover el crecimiento y la regeneración. Las prácticas relacionadas con el aprovechamiento son adoptadas con mayor frecuencia en Bolivia que en Brasil o Perú; entre ellas, la protección de los árboles semilleros, la tala direccional, el monitoreo del crecimiento del bosque, la planificación de las viales de arrastre y el arrastre controlado. Los análisis revelaron que, generalmente, la regulación juega un papel crítico en promover la adopción de mejores prácticas de manejo forestal.

\footnotetext{
' Centro Internacional de Investigación Forestal (CIFOR), Oficina Regional para América Latina. Belém, Brasil. Email: c.sabogal@cgiar.org

${ }^{2}$ Instituto Internacional de Recursos Genéticos Vegetales (IPGRI). Roma, Italia. Email: I.snook@cgiar.org

${ }^{3}$ Consultor independiente. Massachussets, USA. Email: Marco_Boscolo@harvard.edu

${ }^{4}$ Instituto de Silvicultura, Universidad de Freiburg. Freiburg. Alemania. Email: benno.pokorny@waldbau.uni-freiburg.de

${ }^{5}$ Instituto do Homem e o Meio Ambiente da Amazônia. Belém, Brasil. Email. lentini@imazon.org.br

${ }^{6}$ Universidad Autónoma Gabriel René Moreno (UAGRM). Santa Cruz, Bolivia. Email: Iquevedo@scbbs.com.bo

${ }^{7}$ CIFOR, Oficina de Coordinación en Perủ. Pucallpa, Perú. Email: cifor-peru@cgiar.org
} 
En Bolivia, el nivel promedio de adopción aumenta también con una mayor simplicidad (y menores costos de aplicación) de los elementos. En el Perú, la adopción disminuye cuando los beneficios económicos de las prácticas son a largo plazo, su aplicación requiere de altos costos de inversión y las normas no son exigentes. De otro lado, el análisis de la variabilidad en la adopción de prácticas entre las empresas en Brasil mostró que las localizadas en las llamadas fronteras forestales antiguas (con más de 20 años de actividad maderera industrial) están adoptando más las buenas prácticas de manejo que sus similares de fronteras recientes. Al parecer, esta mayor tasa de adopción en las fronteras antiguas no se debe tanto a la mayor experiencia de la empresa en el negocio forestal sino a otros factores, como mejor acceso a información, mayor percepción de la escasez del recurso forestal y mayor presión por cumplir las regulaciones. Además, se encontró que la superficie del área aprovechada anualmente, las inversiones realizadas, el número de trabajadores especializados y bien entrenados y la certificación contribuyen a una mayor adopción. En Bolivia, las inversiones en la concesión (en particular las conducentes a la certificación) influyen en los mayores niveles de adopción. La capacitación periódica a los trabajadores forestales y la asistencia técnica se asocian con una mayor adopción de ciertas prácticas de manejo. En el caso peruano, se encontró una alta variabilidad en la adopción de las prácticas entre pequeños extractores y empresas. Aparte de la obligatoriedad legal, otros factores que afectan la adopción son el desconocimiento de la rentabilidad del manejo, la débil administración de los recursos por el Estado (escasa supervisión y control) y la competencia desleal por madera extraida informal o ilegalmente.

En cada pais se identificó estrategias especificas para mejorar la adopción del MFS en escala empresarial. Entre las estrategias en común destacan una revisión de la normativa forestal (para simplificar y hacer más claras las normas existentes y describir explícitamente cuáles prácticas importantes deben ser implementadas); mayor capacitación técnica a los diferentes actores involucrados en el manejo (desde gerentes hasta trabajadores); incentivos especificos para el manejo forestal (mayor acceso a información, asistencia técnica de calidad, créditos más flexibles); mejora de los sistemas de monitoreo y control; fortalecimiento de la administración forestal y de las instituciones de investigación y extensión forestal.

Palabras claves: Criterios e indicadores; empresas forestales; marco legal. 


\section{CONSTRAINTS AND OPPORTUNITIES TO THE ADOPTION OF SUSTAINABLE FOREST MANAGEMENT PRACTICES IN AMAZONIA RESEARCH RESULTS FROM BRAZIL, BOLIVIA, AND PERU}

\section{SUMMARY}

In spite of the huge investments in research, technical assistance, and training, there is still little adoption of sustainable forest management practices (SFM) in the tropics. In Amazonia, far more knowledge has been generated than is currently applied in forest management for timber production. In 2001, a research project was initiated in Bolivia, Brazil and Peru aiming to analyze the adoption of a set of key practices for SFM by timber enterprises. The project was seeking to determine the factors influencing the adoption of such practices. Based on the results and lessons learned, the project defined strategies to increase the adoption of industrialscale sound management practices. Several methodologies were employed to pursue this goal: a review of the legal framework for forest management in each country; interviews to managers and owners of enterprises and forest professionals working with them (94 enterprises in Brazil, 23 concession-holders in Bolivia, 66 timber producers in Peru), field visits to evaluate the application of the selected key practices (Brazil: 27, Bolivia: 11, Peru: 13), and workshops to discuss the results with a diverse stakeholders of the forest sector.

We found that the rates of adoption of the management practices among the three countries vary considerably. In Brazil, the mean rate of adoption was $56 \%, 57 \%$ in Bolivia, and $41 \%$ in Peru. Practices related to planning are more widely adopted in Brazil than in Bolivia or Peru, though. These practices include the respect of ecological reserves or permanent protection areas, hunting control, timber stock survey, protection of the management area, road planning, protection of future harvest trees, vine cutting, and silvicultural treatments to promote growth and regeneration. Other practices (mainly those related to harvesting) are more frequently adopted in Bolivia than in Brazil or Peru; among them, protection of seed trees, directional felling, monitoring of forest growth, skid trail planning, and controlled skidding. The analysis revealed that, in general, regulation plays a critical role in promoting the adoption of improved forest management practices. In Bolivia, simpler and cheaper to implement practices are better accepted. In Peru, adoption decreases if benefits are long-term, investment costs are high, and regulations are non-mandatory. In Brazil, on the other hand, enterprises located on the 'old forest frontiers' (with more than 20 years of industrial-scale timber activity) are more likely adopters of good forest management practices than their counterparts located in "new forest frontier' areas. Apparently, this wider adoption rate in old frontiers does not have to do with the larger experience of enterprises, but with other factors, such as better access to information, increased perception of forest resource scarcity, and more effective law enforcement.

Other aspects that contribute to a larger adoption of the selected practices are the size of the annual harvesting area, the resources invested, the number of skilled and well trained forest workers, and certification. In Bolivia, investment in concessions (particularly those certification-oriented) influences on higher levels of adoption. Periodical training to workers and technical assistance are also related to a higher adoption of some management practices. In Peru, a high variability between small-scale loggers and timber enterprises adopting management practices was found. Besides legal enforcement, other issues affecting adoption 
are: little knowledge on management profitability, governance failures (lack of supervision and control), and unfair competition from illegal loggers. In each one of the countries evaluated, specific strategies to improve industrial-scale SFM adoption were identified. Some of them, nonetheless, are common to the three countries. Among them, a review of forest regulations (seeking simplification and clarification of existing laws, and explicitly stating the practices to be implemented); more opportunities for training to all stakeholders involved in forest management (from managers to field workers); specific incentives for forest management (e.g. better access to information, quality technical assistance, more flexible access to credit); improved monitoring and control systems; strengthening of the forest administration and of research and outreach agencies.

Key words: Criteria and indicators; forest enterprises; legal framework. 


\section{INTRODUCCIÓN}

Un buen manejo forestal puede aumentar los rendimientos de productos y servicios de un área determinada de bosque y reducir los impactos negativos de la extracción de madera (Bruijnzeel y Critchley, 1994, Fimbel et al., 2001, Putz et al., 2002, Applegate et al., 2004). A pesar de ello, se ha estimado que apenas una minima proporción de los bosques tropicales destinados a fines de producción son manejados con el objetivo de sostener los rendimientos de madera en escala comercial/industrial junto con otros valores y servicios (Poore et al., 1989, FAO, 2005, ITTO, 2006)' ', lo que confirma que la vasta experiencia en manejo de bosques tropicales aún no es aplicada (p.ej., Poore et al., 1989, Higman et al., 1999, Putz et al., 2000).

Aún aquellas prácticas de manejo que son conocidas y han demostrado ser efectivas para reducir el daño causado por el aprovechamiento comercial de madera y para sostener los rendimientos de madera y otros recursos bióticos, se aplican en apenas una pequeña minoria de unidades de aprovechamiento en los bosques tropicales (FAO, 1998, 2001; Richards, 2000, ITTO, 2006).

Dado el interés y las inversiones hechas para promover mejores prácticas en los trópicos, resulta sorprendente lo poco que se conoce actualmente acerca de las limitaciones para la adopción del manejo forestal sostenible (MFS). Hasta la fecha, los debates se han enfocado en percepciones sobre la desventaja financiera asociada con el buen manejo forestal, comparada con la explotación maderera (p.ej., Barreto et al., 1998, Pearce et al., 2001, Rice et al., 2001, Holmes et al., 2002), pero sólo unos cuantos trabajos han analizado los comportamientos y razonamientos de los que manejan los bosques en situaciones específicas (Putz et al., 2000, Karsenty, 2001, Blate et al., 2001).

A fin de entender mejor el grado en el cual las prácticas de MFS son aplicadas en bosques tropicales y los factores que impiden o favorecen su implementación, el Centro Internacional de Investigación Forestal (CIFOR) llevó a cabo un proyecto de investigación entre los años 2001 y 2004 para analizar el nivel de adopción de un conjunto de prácticas claves de MFS por empresas madereras en tres paises amazónicos. Uno de los objetivos era evaluar los impactos de la reforma forestal en Bolivia, iniciada en 1996 con una nueva ley forestal (p.ej. Contreras y Vargas, 2001) que produjo cambios significativos en el contexto institucional para el manejo de sus bosques. Como parte de esta evaluación, se hizo una comparación semejante en Brasil y Perú.

\footnotetext{
' Según un estudio de la FAO (Loyche-Wilkie 2001), la superficie boscosa bajo planes de manejo llega apenas a un 6\% de la cobertura boscosa mundial. En un reciente informe de la Organización Internacional de Maderas Tropicales (OIMT) sobre sus paises miembros productores, se estima que al menos 25,2 millones de hectáreas del área permanente de bosque natural para producción (de madera) - o sea $7,1 \%$ de un total de 353 millones de hectáreas - son manejadas sosteniblemente. Este porcentaje baja a un 3,5\% (6,47 millones de hectáreas de un total de 185 millones) en el caso de países productores en América Latina y el Caribe (ITTO 2006).

${ }^{2}$ Para Bolivia: Snook et al. (en prensa); para Brasil: Sabogal et al. (2006) y Sabogal et al. (en prensa); para Perú: Smith et al. $(2003,2006)$, Colán et al. (en prensa).
} 
La investigación fue diseñada para responder a las siguientes dos preguntas principales: (1) ¿En qué grado empresas que manejan áreas boscosas en estos tres países aplican buenas prácticas de manejo para sostener la productividad y los servicios ambientales del bosque?; y (2) ¿Cuáles son los factores que influencian (restringen o fomentan) la adopción de estas prácticas? Uno de los propósitos del estudio fue usar los resultados para proponer estrategias y recomendaciones especificas para aumentar la adopción de buenas prácticas de manejo por empresas forestales en la Amazonia.

\section{ANTECEDENTES}

\section{Recurso Forestal y Marco Legal para el Manejo del Bosque Amazónico en los Países de Estudio}

El Cuadro $\mathrm{N}^{\circ} 1$ son presentadas varias estadisticas sobre cobertura forestal y producción de madera para los tres países. El recurso forestal cubre más de la mitad de la superficie en cada país, localizado mayormente en la porción amazónica. Según un reciente informe de la OIMT (ITTO, 2006), los bosques naturales con fines de producción representan, en términos de cobertura forestal total, alrededor de $29 \%$ en Bolivia, $21 \%$ en Brasil y $36 \%$ en Perú. Además, los tres paises presentan superficies comparativamente similares (entre 5,0 y 5,47 millones ha) de bosques naturales con planes de manejo. En términos relativos, sin embargo, Brasil tiene solamente $5,4 \%$ de su área forestal permanente de producción natural bajo planes de manejo, en comparación con $20 \%$ en el Perú y $32 \%$ 'en Bolivia. De otro lado, con 2,21 millones ha, este último país tiene la superficie más grande de bosque natural certificado en América Latina, habiendo conseguido un alto estándar de manejo forestal.

\section{AREAS FORESTALES Y PRODUCCIÓN DE MADERA EN LOS BOSQUES AMAZÓNICOS DE BOLIVIA, BRASIL Y PERÚ}

\begin{tabular}{|c|c|c|c|}
\hline Estadisticas & Bolivia & Brasil & Perú \\
\hline $\begin{array}{l}\text { 1. Area forestal total, en } 1000 \text { ha (en paréntesis el porcentaje de la } \\
\text { superficie total del pais) }\end{array}$ & $\begin{array}{r}58740 \\
(54.2 \%)\end{array}$ & $\begin{array}{l}477698 \\
(57.2 \%)\end{array}$ & $\begin{array}{r}68742 \\
(53.7 \%)\end{array}$ \\
\hline $\begin{array}{l}\text { 2. Tasa anual de deforestación para el periodo } 2000-2005 \text {, en } \\
\text { ha/año (en paréntesis el porcentaje del cambio anual total) }\end{array}$ & $\begin{array}{r}-135200 \\
(-0.5 \%) \\
\end{array}$ & $\begin{array}{r}-3466000 \\
(-0.6 \%)\end{array}$ & $\begin{array}{r}-224600 \\
(-0.1 \%) \\
\end{array}$ \\
\hline \multicolumn{4}{|l|}{ 3. Área Forestal Permanente (AFP), en 1000 ha } \\
\hline Total & 31760 & 372810 & 41150 \\
\hline Producción natural & 17000 & 98100 & 24600 \\
\hline Adjudicado a concesiones/bajo licencia & 5470 & n.a. & 8000 \\
\hline Con planes de manejo & 5470 & 5250 & 5000 \\
\hline Certificado & 2210 & 1160 & $58.5^{*}$ \\
\hline Manejado sosteniblemente & 2210 & 1360 & 560 \\
\hline \multicolumn{4}{|l|}{ 4. Producción nacional de madera industrial en 2003, en $\mathrm{m}^{3}$} \\
\hline Madera redonda (trozas) & 650000 & 29700000 & 1290000 \\
\hline Madera aserrada (maderas tropicales) & 347000 & 15900000 & 528000 \\
\hline Madera compensada (maderas tropicales) & - & 1200000 & 101000 \\
\hline 5. Número de empresas madereras & $84^{4}$ & $2146^{\circ}$ & $584^{2}$ \\
\hline
\end{tabular}

Fuentes de información para los items 1 y 2: FAO (2005); para los items 3 y 4: ITTO (2006).

- Número de concesiones al año 2003. Fuente: Superintendencia Forestal (2004).

- Número de empresas madereras en la Amazonia Legal en el 2004, sin contar los micro-aserraderos (con consumo de 1500 $\mathrm{m}^{3}$ de trozas/año). Fuente: Lentini et al., 2005.

' Número de contratos en las concesiones forestales con fines maderables, sobre una superficie total de 7,38 mill. ha. Fuente: INRENA, 2006. En comparación, hasta mediados del 2001 se tenian 1,85 mill. ha de bosques en explotación bajo el régimen forestal anterior a través de más de 1500 contratos de aprovechamiento, la mayoria en áreas por debajo de 1000 ha, con un volumen total autorizado de madera bajo esos contratos de unos 3 millones $\mathrm{m}^{3}$ (INRENA, 2001). 
Con respecto al marco legal, en Bolivia el manejo forestal es regulado por la Ley Forestal 1700 de Julio de 1996 y su reglamento aprobado en diciembre de 1996 y una serie de normas técnicas aprobadas en 1997. La mayoria de los bosques naturales pertenecen al Gobierno. El acceso a los recursos forestales se basa en permisos de aprovechamiento de madera que pueden ser adquiridos legalmente por: a) Empresas madereras por medio de concesiones en terrenos nacionales; b) Asociaciones Locales del Lugar, por medio de concesiones sobre terrenos municipales; c) Permisos de manejo forestal en terrenos indigenas (Tierras Comunitarias de Origen); o d) Permisos de aprovechamiento en terrenos privados.

Todos estos requieren de la aprobación por parte de la Superintendencia Forestal de un plan de manejo forestal y planes operativos anuales forestales y todos requieren el pago anual de una patente forestal de US\$1/ha (Contreras y Vargas, 2002, Pacheco, 2003) ${ }^{1}$. La Superintendencia Forestal ha gozado de independencia y cierta autonomía económica y administrativa del gobierno central (Cámara Forestal de Bolivia, 2006).

El sector forestal del Brasil es fuertemente regulado, si bien las regulaciones han evolucionado parcialmente reflejando esfuerzos del gobierno para incorporar el creciente conocimiento ecológico y social acerca de sus bosques tropicales (Lele et al., 2000). La ley forestal enmendada de 1965 (Código Florestal, Lei 4.771/65) es el principal instrumento de política forestal en el país, estableciendo la necesidad de planes de manejo para el aprovechamiento de recursos forestales en la Amazonia. Pero fue sólo en 1994 que el gobierno definió el MFS en términos prácticos (Hummel, 2000) a través de instrucciones normativas que disciplinaban su aplicación para la elaboración y presentación de planes de manejo forestal. Está en vigor la norma MMA 04/ 2002 (IN-04), que establece las normas técnicas para el manejo forestal en la Amazonia. Por ley, el uso de los recursos naturales forestales en tierras privadas requiere la presentación de un plan de manejo forestal. También puede aprovecharse madera cuando la tierra es convertida a otros usos tales como la agricultura. Casi todo el manejo para producción es conducido por empresas privadas en bosques bajo propiedad privada (ITTO, 2006) ${ }^{2}$.

\footnotetext{
' Debido a la crisis económica del sector forestal, en 2003 se cambió el sistema de pago de patente a uno mucho más favorable a las empresas concesionarias, de la siguiente manera: US\$1/ha efectivamente aprovechada (es decir, por el área de aprovechamiento anual), más aproximadamente US\$4/ha por concepto de patente de regulación (en beneficio de la Superintendencia Forestal). El monto total a pagar es entonces de aproximadamente US $\$ 5 /$ ha efectivamente aprovechada (Camacho, 2003).

${ }^{2}$ El Ministerio del Medio Ambiente (MMA) viene conduciendo desde el 2003 un proceso de reformulación de los reglamentos que interfieren en el manejo forestal en la Amazonia, en dos líneas o ejes. El primero y más importante ha sido la formulación y aprobación (en marzo del 2006) de una ley para la gestión de los bosques públicos que, entre otros, establece reglas para el manejo forestal en áreas públicas por medio de tres modalidades (PNF). El segundo eje es la revisión de las normas para el manejo forestal: en la actual normativa la diferenciación de las categorias de planes de manejo forestal está fuertemente influenciada por el perfil del ejecutor (razón social, organización social), pero que poco se relaciona a exigencias técnicas compatibles con su real capacidad técnica de ejecutar el manejo forestal (Freitas y Hummel, en prensa).

${ }^{3}$ Como parte de dicha ley, el aprovechamiento de maderas en el pais se venia realizando a través de tres modalidades: permisos, autorizaciones y contratos. Habían dos tipos de contratos: aquellos para superficies mayores de 1000 ha y hasta 100,000 ha por 40 años renovables cada 10 años, que exigían la presentación de estudios de factibilidad técnicoeconómica. El otro tipo eran contratos de corta duración (de dos hasta máximo 10 años) sobre superficies menores a 1000 ha, que fueron diseñados como respuesta social para los pequeños extractores que empleaban prácticas de extracción no mecanizada; entre otras facilidades, en este tipo de contrato no se exigían planes de manejo o estudios especificos. Esta opción constituyó la fórmula ideal que emplearon muchos industriales y acopiadores de madera para burlar las exigencias de planes de manejo, pues resultaba más fácil capitalizar a los pequeños extractores o contratarlos como mano de obra para manejar varios contratos de menos de 1000 ha que la autorización para extraer y producir en varios miles de hectáreas, lo cual requeria de estudios específicos (Hidalgo 2003).
} 
En el caso del Perú, el principal instrumento legal es la Ley Forestal y de Fauna Silvestre 27308 del 2000 y su reglamento aprobado en 2001 . Esta nueva ley reemplazó a la anterior de 1975 (Ley Forestal y de Fauna Silvestre, Decreto Ley $N^{\circ} 21147$ ), que creó un virtual caos en la explotación de los recursos forestales más accesibles en la Amazonia y un sistema con extensos impactos sociales (p.ej., Smith et al. 2003, Galarza y La Serna 2005) ${ }^{3}$. Sin embargo, durante el periodo del estudio estaba vigente todavia la ley anterior, que daba acceso a los bosques. Con la promulgación de la Ley 27308 se inició un nuevo régimen forestal que promueve el uso integral del recurso forestal y compromete la elaboración e implementación de planes de manejo para todas las modalidades de acceso al recurso.

\section{MÉTODOS}

El estudio se llevó a cabo entre los años de 2001 y 2004 a través de tres fases, cada una aplicando metodologias específicas: 1) Entrevistas con empresarios (fase 1); 2) Evaluaciones de campo (fase 2), y 3) Talleres de discusión con diferentes actores (fase 3). El foco del estudio'se'fue centrado en las principales áreas productoras de madera en las zonas tropicales de Bolivia (Departamentos de Santa Cruz, Beni, Pando y La Paz), Brasil (Estados de Pará, Mato Grosso y Rondônia) y Perú (Departamentos de Ucayali y Loreto).

Para preparar el estudio se recopiló información secundaria y se estableció contactos con diversos actores claves en los tres paises. Además, se realizó una calificación preliminar de los factores macro que afectan la adopción del manejo forestal y se diseñó el marco conceptual. También se llevó a cabo un análisis comparativo del sector forestal empresarial y del marco legal/regulador para el manejo forestal en los tres paises. Finalmente, se seleccionó las prácticas de MFS a ser evaluadas durante el estudio y las empresas forestales a ser entrevistadas

\section{Definición de Prácticas de Buen Manejo}

Se evaluó hasta qué grado se estaba aplicando un conjunto de prácticas "claves", que contribuyen a la sostenibilidad del recurso forestal. Se seleccionó un conjunto de 14 prácticas de buen manejo forestal (Cuadro $\mathrm{N}^{\circ} 2$ ) con base en consultas a varias fuentes, principalmente los requerimientos de los principios y criterios del FSC y los lineamientos de la OIMT, así como Dykstra y Heinrich (1996), Higman et al. (1999), Blakeney et al. (1998), Hammond et al. (2000) y Sabogal et al. (2000). Cada una de estas prácticas de MFS contribuye a sostener o incrementar la productividad maderable del bosque; al mismo tiempo, la mayor parte rinde otro(s) beneficio(s) socioeconómicos al productor, entre ellos: aumento de la eficiencia de las operaciones y reducción de sus costos o aumento de la seguridad física de los trabajadores. Los beneficios derivados de las diferentes prácticas se logran en diferentes momentos del tiempo (por ejemplo, durante la extracción actual, en el siguiente ciclo, o más allá del siguiente ciclo). Algunas prácticas rinden beneficios a la sociedad, los cuales no son captados por el productor (por ejemplo, el control de la caceria y la protección de áreas de reserva, que mantienen poblaciones de plantas y animales que contribuyen a la polinización y dispersión de semillas y a otros procesos ecológicos). 


\section{Cuadro $\mathrm{N}^{\circ} 2$}

PRÁCTICAS DE BUEN MANEJO FORESTAL, SUS BENEFICIOS Y EL PLAZO DEL BENEFICIO

\begin{tabular}{|c|c|c|c|c|}
\hline \multicolumn{2}{|r|}{ Prácticas de MFS } & \multirow{2}{*}{\begin{tabular}{|l|} 
Contribución a la Sostenibilidad \\
Herramienta para la planifi- \\
cación de brechas/ pistas \\
de extracción para reducir \\
daños y aumentar eficien- \\
cia
\end{tabular}} & \multirow{2}{*}{\begin{tabular}{|l|}
\multicolumn{1}{|c|}{ Otro Beneficio } \\
Información útil para la \\
planificación y el merca- \\
deo
\end{tabular}} & \multirow{2}{*}{\begin{tabular}{l}
\multicolumn{1}{c}{ Plazo del Beneficio } \\
Aprovechamiento \\
actual (y subsecuente, si \\
se incluyen árboles de \\
futura cosecha)
\end{tabular}} \\
\hline 1 & $\begin{array}{l}\text { Censo y mapeo de árboles } \\
\text { comerciales }\end{array}$ & & & \\
\hline 2 & $\begin{array}{l}\text { Corta de lianas en árboles } \\
\text { a extraer ( } 6 \text { meses antes } \\
\text { de la zafra) }\end{array}$ & $\begin{array}{l}\text { Reducción de daños al árbol } \\
\text { a cortar y a árboles vecinos }\end{array}$ & $\begin{array}{l}\text { Reducción de riesgos al } \\
\text { cortador }\end{array}$ & $\begin{array}{l}\text { Aprovechamiento } \\
\text { actual }\end{array}$ \\
\hline 3 & $\begin{array}{l}\text { Planificación, construcción y } \\
\text { mantenimiento de la } \\
\text { infraestructura vial }\end{array}$ & $\begin{array}{l}\text { Reducción de daños a sue- } \\
\text { los y ríos; aumento en la } \\
\text { eficiencia de la extracción } \\
\text { y el manejo }\end{array}$ & $\begin{array}{l}\text { Reducción de tiempo } \\
\text { y costo de transporte; } \\
\text { reducción en costo de } \\
\text { reparación de vehiculos }\end{array}$ & $\begin{array}{l}\text { Aprovechamiento } \\
\text { actual }\end{array}$ \\
\hline 4 & Planificación del arrastre & $\begin{array}{l}\text { Reducción de daños a } \\
\text { suelos, rios y árboles de } \\
\text { futura cosecha }\end{array}$ & $\begin{array}{l}\text { Aumento en eficiencia; re- } \\
\text { ducción en costos de ex- } \\
\text { tracción }\end{array}$ & $\begin{array}{l}\text { Aprovechamiento } \\
\text { actual }\end{array}$ \\
\hline 5 & Corte direccional ${ }^{+}$ & $\begin{array}{l}\text { Reducción de daños al árbol } \\
\text { a cortar; protección de árbo- } \\
\text { les vecinos }\end{array}$ & $\begin{array}{l}\text { Mayor eficiencia en la } \\
\text { extracción }\end{array}$ & $\begin{array}{l}\text { Aprovechamiento } \\
\text { actual y futuros }\end{array}$ \\
\hline 6 & $\begin{array}{l}\text { Protección de árboles de } \\
\text { futura cosecha (incluyendo } \\
\text { el corte direccional y la corta } \\
\text { de lianas)* }\end{array}$ & $\begin{array}{l}\text { Protección de la producción; } \\
\text { aumento del crecimiento } \\
\text { (por la corta de lianas) }\end{array}$ & $\begin{array}{l}\text { Aumento en volumen } \\
\text { aprovechable }\end{array}$ & $\begin{array}{l}\text { Próximo } \\
\text { aprovechamiento }\end{array}$ \\
\hline 7 & $\begin{array}{l}\text { Protección de árboles } \\
\text { semilleros (incluyendo } \\
\text { el corte direccional) }\end{array}$ & $\begin{array}{l}\text { Favorecimiento de la } \\
\text { regeneración de espe- } \\
\text { cies deseadas }\end{array}$ & $\begin{array}{l}\text { Árboles semilleros dejados } \\
\text { proveen volumen para el } \\
\text { próximo aprovechamiento }\end{array}$ & $\begin{array}{l}\text { Próximo } \\
\text { aprovechamiento y } \\
\text { más allá }\end{array}$ \\
\hline 8 & Arrastre de bajo impacto & $\begin{array}{l}\text { Reducción de daños a } \\
\text { suelos, rios y vegetación }\end{array}$ & $\begin{array}{l}\text { Aumento en eficiencia de } \\
\text { extracción reduce costos }\end{array}$ & $\begin{array}{l}\text { Aprovechamiento } \\
\text { actual y futuros }\end{array}$ \\
\hline 9 & $\begin{array}{l}\text { Establecimiento y respeto de } \\
\text { zonas de protección (dentro } \\
\text { del área de manejo; p.ej. ribe- } \\
\text { ras de rios, áreas empinadas) }\end{array}$ & $\begin{array}{l}\text { Protección de la biodiversidad } \\
\text { y los servicios ecológicos que } \\
\text { mantienen la productividad }\end{array}$ & $\begin{array}{l}\text { Sostenibilidad de la pro- } \\
\text { ducción; mayores opcio- } \\
\text { nes futuras }\end{array}$ & $\begin{array}{l}\text { Más allá del } \\
\text { próximo } \\
\text { aprovechamiento }\end{array}$ \\
\hline 10 & $\begin{array}{l}\text { Protección del área de mane- } \\
\text { jo (contra incendios, invasio- } \\
\text { nes, etc.) }\end{array}$ & $\begin{array}{l}\text { Mantenimiento del recurso } \\
\text { forestal }\end{array}$ & $\begin{array}{l}\text { Mantenimiento de la pro- } \\
\text { ducción a largo plazo }\end{array}$ & $\begin{array}{l}\text { Corto, mediano y } \\
\text { largo }\end{array}$ \\
\hline 11 & Control de la caza & $\begin{array}{l}\text { Protección de la biodiversidad } \\
\text { y los servicios ecológicos que } \\
\text { mantienen la regeneración y } \\
\text { la productividad }\end{array}$ & $\begin{array}{l}\text { Sostenibilidad de la pro- } \\
\text { ducción; mayores op- } \\
\text { ciones futuras }\end{array}$ & $\begin{array}{l}\text { Más allá del } \\
\text { próximo } \\
\text { aprovechamiento }\end{array}$ \\
\hline 12 & $\begin{array}{l}\text { Monitoreo del crecimiento } \\
\text { del bosque }\end{array}$ & $\begin{array}{l}\text { Herramienta para mantener } \\
\text { rendimientos al asegurar el } \\
\text { reemplazo de volúmenes } \\
\text { aprovechados }\end{array}$ & $\begin{array}{l}\text { Mantenimiento de } \\
\text { volúmenes de apro- } \\
\text { vechamiento }\end{array}$ & $\begin{array}{l}\text { Próximo } \\
\text { aprovechamiento y } \\
\text { más allá }\end{array}$ \\
\hline 13 & $\begin{array}{l}\text { Prácticas de silvicultura para } \\
\text { favorecer el crecimiento } \\
\text { (incluyendo corta de lianas } \\
\text { en árboles de futura cosecha) }{ }^{++}\end{array}$ & $\begin{array}{l}\text { Aumento en la tasa de } \\
\text { producción y su calidad }\end{array}$ & $\begin{array}{l}\text { Mayores volúmenes/ ta- } \\
\text { maños de especies e } \\
\text { individuos deseados }\end{array}$ & $\begin{array}{l}\text { Próximo } \\
\text { aprovechamiento }\end{array}$ \\
\hline 14 & $\begin{array}{l}\text { Prácticas de silvicultura post- } \\
\text { aprovechamiento para ase- } \\
\text { gurar la regeneración }{ }^{* *}\end{array}$ & $\begin{array}{l}\text { Asegura la regeneración de } \\
\text { especies deseadas }\end{array}$ & $\begin{array}{l}\text { Mayores volúmenes } \\
\text { de especies deseadas }\end{array}$ & $\begin{array}{l}\text { Más allá del } \\
\text { próximo } \\
\text { aprovechamiento }\end{array}$ \\
\hline
\end{tabular}

,+++ En la primera etapa del estudio, las prácticas con los mismos símbolos fueron combinadas en una sola. 


\section{Entrevistas con Empresarios (Fase 1)}

Entre el 2001 y el 2002 se realizó entrevistas al propietario o gerente de la empresa y al encargado técnico (generalmente un profesional o técnico forestal) de las operaciones forestales, con el fin de caracterizar la empresa y conocer las percepciones de ambos actores sobre las prácticas de manejo y sobre los principales factores económicos, legales e institucionales que facilitaban o limitaban su adopción por la empresa. Para ello se aplicaron dos cuestionarios: (1) del empresario se colectó información a nivel de la empresa y sus percepciones sobre la deseabilidad de las prácticas individuales, mientras que (2) del encargado técnico se colectó información sobre cuáles prácticas eran adoptadas y cómo eran implementadas en el campo'.

Las empresas fueron seleccionadas al azar a través de un muestreo estratificado por departamento/estado y, dentro de estos, por región o zona maderera. En Bolivia, la estratificación dentro de los departamentos se hizo por región (Choré, Chiquitania, Guarayos, Bajo Paragua y Amazonia), entrevistándose al $30 \%$ de los concesionarios en cada departamento, resultando en un total de 23 concesiones controladas por 20 empresas (mayores detalles en Snook et al., en prensa). En Brasil, la estratificación para el muestreo se basó en las llamadas "micro-regiones" pertenecientes a diferentes zonas de frontera maderera ${ }^{2}$, llegándose a entrevistar 94 empresas localizadas en-áreas de frontera forestal vieja, intermedia y nueva en los tres estados (para mayores detalles, ver Sabogal et al., 2005, 2006). En el caso del Perú, se entrevistó a $10 \%$ de los extractores con contratos pequeños (superficies $<1000$ ha) y a todas las empresas con contratos grandes (8), totalizando 66 entrevistas en los dos departamentos. Los contratistas evaluados consistieron de 34 pequeños extractores que seguian prácticas manuales de extracción y 32 empresas madereras que usaban predominantemente la extracción mecanizada (mayores detalles en Smith et al., 2003, Colán et al., en prensa).

\section{Evaluaciones de Campo (Fase 2)}

Durante las épocas de zafra en el 2002 y el 2003 se llevó a cabo visitas de campo a las zonas de extracción de una sub-muestra de empresas que habian sido parte de las entrevistas en la primera fase del estudio. Esta fase tenía dos objetivos: evaluar hasta qué grado las respuestas a las entrevistas reflejaban la forma de trabajo en el bosque, y permitir un análisis más a fondo de los incentivos y las limitantes a la adopción de las prácticas de MFS, tomando en cuenta las perspectivas de los trabajadores que las aplican. Se realizó entrevistas con las

\footnotetext{
' Se tuvieron algunas diferencias en la metodologia seguida entre los países en función a las distintas situaciones e intereses. En Bolivia, la entrevista se complementó con información respecto al tratamiento de la práctica por la ley y con una evaluación categórica si la práctica, en caso que sea obligatoria por ley, era fácil de hacerse cumplir. En Brasil, el cuestionario al forestal se aplicó solamente en el estado de Pará, no continuándose en los otros dos estados debido a que resultó evidente que las informaciones que estos proporcionaron sobre la aplicación de las prácticas de buen manejo no correspondian con la realidad conocida. En Perú no se incluyó dicho cuestionario porque la mayor parte de productores no contaba con un profesional o técnico forestal.

${ }^{2}$ El concepto de fronteras forestales lleva en consideración los diferentes tipos de bosque (abierto y denso), el tiempo desde la abertura de la frontera y las condiciones de acceso a los recursos forestales y la infraestructura (caminos, rios) (Veríssimo et al. 2002).
} 
personas a cargo de las operaciones de aprovechamiento y se colectó las percepciones de los operadores (motosierristas, tractoristas) acerca de las prácticas de manejo forestal. La evaluación incluyó la verificación de documentos tales como planes de manejo forestal y planes operacionales anuales, mapas, libretas de campo y formatos de registro de informaciones, además del levantamiento de observaciones y datos en el bosque. En Bolivia se evaluó 11 concesiones de las 23 entrevistadas, en Brasil fueron 27 empresas (un 29\% de las inicialmente entrevistado) y en Perú 13 contratistas que tenian área de extracción autorizada bajo el anterior régimen forestal (Smith et al., 2006)'.

Para poder evaluar el grado de adopción de cada una de las prácticas de buen manejo seleccionadas, cada práctica fue subdividida en elementos, concebidos como indicadores o componentes que eran posibles de evaluar a través de preguntas u observaciones ${ }^{2}$. Cada práctica consistia de entre uno a ocho elementos, para un total de 77 elementos que sirvieron para el análisis comparativo posterior ${ }^{3}$. Para reducir el alto número de elementos y facilitar asi los análisis posteriores, se introdujo el concepto de elemento "crítico", entendido como aquel que, siendo bien aplicado, podria lograr la mayoria de beneficios de la práctica ${ }^{4}$. Se analizó el cumplimiento de 52 elementos considerados críticos (con valor 1).

Para cada uno de los elementos el estudio intentó determinar las siguientes preguntas: (i) ¿cuáles elementos de las prácticas son adoptados?. (ii) ¿por qué están siendo adoptados algunos elementos y otros no?; y (iii) ¿por qué algunas empresas están adoptando buenas prácticas (el conjunto o la mayoria de los elementos de la misma) y otras no?.

La primera pregunta se contestó en base a las entrevistas y observaciones de campo. El grado de adopción de una práctica de manejo se determinó sumando las observaciones sobre el cumplimiento o no de los diferentes elementos de la práctica. Para cumplir en un $100 \%$ una práctica, habia que llevar a cabo todos los elementos de esta práctica; en caso de cumplir con una proporción de elementos, se calculó que una práctica se estaba llevando a cabo a un nivel porcentual correspondiente a la proporción de elementos de la práctica que se llevaron a cabo.

Para responder a la segunda pregunta, se planteó como hipótesis que varios atributos estarían afectando la probabilidad de adopción: (a) si los beneficios privados se realizaban a corto, mediano o largo plazo, o no habian beneficios privados (H1.a Beneficio económico); (b)

\footnotetext{
' En esta fase se buscó Identificar las prácticas empleadas con cada método de extracción (manual y mecanizado) y las posibles diferencias entre productores, asi como conocer mejor el contexto y las razones de su aplicación o no. Las categorias de productores forestales fueron (Smith et al 2003): los pequeños extractores que administran uno o pocos contratos menores a 1,000 ha; las empresas grandes que administran varios contratos pequeños; $y$ los productores con contratos grandes.

${ }^{2}$ La definición de elementos indicadores de prácticas bien realizadas se basó en la metodologia utilizada por el Proyecto EMBRAPACIFORITTO (Pokorny et al., 2005).

${ }^{3} \mathrm{El}$ número de elementos por país varió ligeramente en función a las condiciones locales. Una lista completa de los elementos evaluados en cada pais se puede ver en Snook et al. en prensa para Bolivia, Sabogal et al., 2005,2006 para Brasil y Colán et al,.en prensa para Perú.

${ }^{4}$ Por ejemplo, en el caso de la práctica Corta de lianas antes del aprovechamiento, se identificaron tres elementos: (1) Las lianas son cortadas, cuando fuese necesario, en los árboles a aprovechar y en los árboles vecinos cuando hubiese entrelazamiento con éstos; (2) Se lleva a cabo la corta de lianas con por lo menos seis meses de anticipación al aprovechamiento; y (3) El corte de lianas es realizado correctamente.
} 
en los tres paises difirieron ligeramente. A fin de mostrar comparaciones que sean consistentes, dicho cuadro presenta las tasas de adopción de prácticas calculadas usando elementos considerados "críticos" en al menos un pais y que habian sido evaluadas en al menos dos paises (Brasil y Bolivia). Esto significa que elementos que habian sido evaluados sólo en Brasil, por ejemplo, no fueron usados para computar las evaluaciones del mencionado cuadro.

En el Cuadro $N^{\circ} 4$ se incluye también las evaluaciones llevadas a cabo en Perú, aún si, como se indicó en Colán et al. (en prensa), los elementos y operadores evaluados diferian significativamente de los otros dos paises.

\section{Cuadro $\mathrm{N}^{\circ} 4$}

TASAS DE ADOPCIÓN (\%) DE PRÁCTICAS CLAVES EN LOS PAISES DE ESTUDIO

\begin{tabular}{|c|c|c|c|c|}
\hline Práctica & $\begin{array}{c}\mathbf{N}^{\circ} \\
\text { Elementos }\end{array}$ & Brasil & Bolivia & Perú \\
\hline 1. Censo y mapeo de árboles comerciales & 8 & 86.7 & 81.8 & $51.7^{*}$ \\
\hline 2. Corta de lianas en árboles a extraer & 3 & 32.7 & 12.1 & $50.0^{*}$ \\
\hline 3. Planificación, construcción y mantenimiento de la infraestructura vial & 17 & 72.8 & 67.5 & $54.9^{*}$ \\
\hline 4. Planificación del arrastre & 7 & 28.6 & 59.7 & $65.0^{*}$ \\
\hline 5. Corte direccional & 13 & 49.1 & 58.0 & $8.6^{*}$ \\
\hline 6. Protección de árboles de futura cosecha & 2 & 68.5 & 31.8 & - \\
\hline 7. Protección de árboles semilleros & 3 & 51.8 & 81.8 & $0.0^{*}$ \\
\hline 8. Arrastre de bajo impacto & 7 & 25.7 & 71.9 & $58.0^{*}$ \\
\hline 9. Establecimiento y respeto de zonas de protección & 5 & 82.2 & 78.2 & $53.3^{*}$ \\
\hline 10. Protección del área de manejo & 3 & 95.1 & 80.3 & 0.0 \\
\hline 11. Control de la caza & 1 & 88.9 & 72.7 & 60.0 \\
\hline 12. Monitoreo del crecimiento del bosque & 4 & 44.9 & 60.2 & 0.0 \\
\hline 13. Prácticas de silvicultura para favorecer el crecimiento & 1 & 25.9 & 9.1 & - \\
\hline 14. Prácticas de silvicultura para asegurar la regeneración & 3 & 14.8 & 3.0 & $\cdot$ \\
\hline
\end{tabular}

- Estos elementos para Perú no son consistentes con los de Brasil y Bolivia.

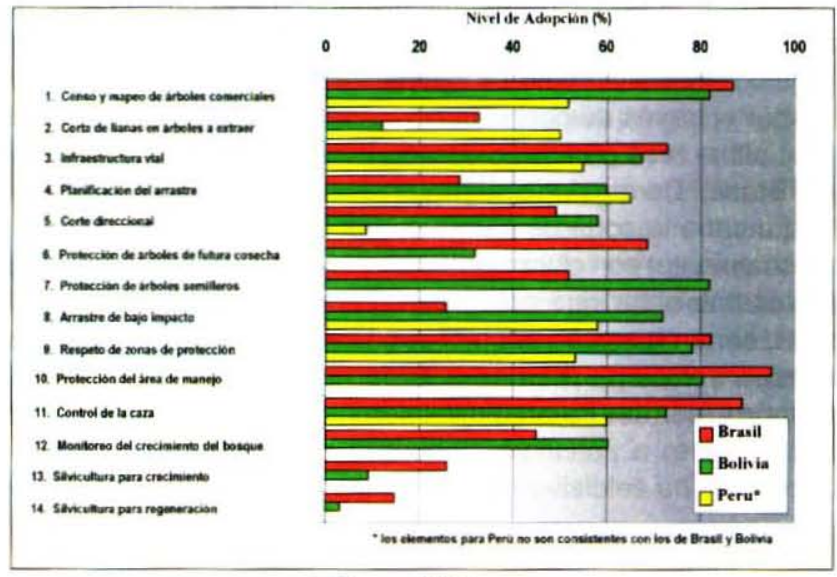

Figura $\mathrm{N}^{\circ} 1$

TASAS DE ADOPCIÓN (\%) DE PRÁCTICAS CLAVES EN LOS PAISES DE ESTUDIO 
Mientras que en los tres paises las tasas de adopción difieren marcadamente entre las prácticas, existen también claras diferencias entre los paises. Por ejemplo, algunas prácticas son adoptadas más ampliamente en Brasil que en Bolivia. El análisis reveló que estas prácticas estaban mayormente relacionadas con la planificación, incluyendo prácticas como : protección de reservas, control de la caza, inventario comercial al $100 \%$, protección del área de manejo, planificación de caminos, protección de árboles de futura cosecha, corta de lianas, tratamientos silviculturales para promover el crecimiento y tratamientos silviculturales para promover la regeneración. Otras prácticas, mayormente relacionadas al aprovechamiento, fueron más adoptadas en Bolivia que en Brasil, incluyendo: protección de árboles semilleros, tala dirigida, monitoreo del crecimiento del bosque, planificación del arrastre y arrastre controlado. Con todo, nueve prácticas fueron adoptadas más frecuentemente en Brasil que en Bolivia y cinco fueron adoptadas más frecuentemente en Bolivia que en Brasil. Algo preocupante con estas cifras son las tasas extremadamente bajas de adopción de prácticas silviculturales en Bolivia $(9,1 \% \text { y } 3 \%)^{\prime}$. Al calcular un promedio simple entre las 14 prácticas, sin embargo, se encuentra que los dos paises tienen una tasa de adopción global muy similar de alrededor de $55 \%$.

¿Qué podría ayudar a explicar esta variabilidad? ¿Por qué algunas prácticas son más adoptadas más frecuentemente en un pais y viceversa? A pesar de que no se contó con suficiente información para investigar rigurosamente las razones para estas diferencias, se pudo calcular la correlación entre la diferencia en las tasas de adopción entre Bolivia y Brasil y la diferencia en la claridad de las regulaciones y en el costo de la adopción (medido por la simplicidad de aplicación de la práctica). Estos análisis se complementaron con algunas hipótesis basadas en observaciones anecdóticas:

Los costos (percibidos) de adopción de las prácticas difieren en los dos países. Donde se percibe que los costos de adopción/inversión son más bajos (para la misma práctica), las tasas de adopción para esa práctica tienden a ser más altas. Esta hipótesis se apoya por lo que fue observado en Bolivia, donde las prácticas que requieren de mayores inversiones fueron adoptadas menos. Como se esperaba, pareceria que la adopción es influenciada por el costo de la mano de obra y el equipo.

Las regulaciones difieren en los dos paises. Por ejemplo, el monitoreo del crecimiento del bosque a través de parcelas permanentes es obligatorio en Bolivia, pero no en Brasil, y alli la tasa promedio de adopción de esta práctica es de $60 \%(15 \%$ más que en Brasil). De otro lado, algunos elementos de la práctica corta de lianas (p.ej. se lleva a cabo la corta de lianas con por lo menos seis meses de anticipación al aprovechamiento) son claramente obligatorios en Brasil, pero no en Bolivia. También es interesante notar que prácticas tales como la protección de árboles de futura cosecha, tienen tasas de adopción más altas en Brasil, mientras que la protección de árboles semilleros tiene tasas de adopción mayores en Bolivia. Estas dos prácticas reciben un tratamiento diferente en la legislación, esto es, en Brasil se 
hace la referencia a "árboles residuales", que incluyen ambas categorias (árboles de futura cosecha y árboles semilleros), y en la legislación no hay una clara definición de lo que es un árbol semillero. Con todo, sin embargo, las diferencias en cuanto a claridad de las regulaciones no se correlacionaron fuertemente con la diferencia en la tasa de adopción entre los dos paises.

Cumplimiento de la ley. Por una variedad de razones, las agencias reguladoras hacen cumplir ciertas prácticas más que otras. En Bolivia, por ejemplo, prácticas tales como la protección de reservas, el inventario comercial al $100 \%$ y la protección de árboles semilleros, han sido enfatizadas por la SF, que considera como muy severas las violaciones a estas regulaciones. En dicho pais estas prácticas están siendo adoptadas con tasas mayores a $80 \%$. Similarmente en Brasil, ciertas prácticas, tales como la protección de reservas (conocidas como áreas de protección permanente), son auditadas por IBAMA muy cuidadosamente en sus inspecciones de campo.

Las caracteristicas del bosque y las prácticas prevalecientes (p.ej., intensidad de aprovechamiento) difieren entre paises, de manera que la importancia de una práctica dada (tanto desde el punto de vista del manejo como del punto de vista de hacer cumplir la ley) también difiere.

Los profesionales forestales juegan papeles diferentes en el manejo forestal en los dos paises. En Brasil, por ejemplo, los profesionales forestales son contratados principalmente por empresas para preparar y someter planes de manejo y realmente no participan (al menos como debiera ser) en su implementación. En Bolivia, de otro lado, los forestales están involucrados en todo el proceso y tienen responsabilidad civil y penal por sus actos profesionales e información que presenten a la Superintendencia Forestal. Como resultado, las diferencias en los niveles de adopción son bastante pequeñas con respecto a los procesos de planificación (p.ej., en el inventario comercial al $100 \%$ y la planificación de caminos), pero son mayores para las operaciones de aprovechamiento tales como la planificación del arrastre y el arrastre controlado.

\section{Variabilidad Entre Elementos Indicadores de Prácticas Bien Realizadas}

Los análisis a nivel de pais empezaron atendiendo a la pregunta: ¿Se están adoptando las prácticas con la misma probabilidad? 'Si no, ¿por qué es más probable que se adopten algunas prácticas más que otras? Los análisis revelaron que generalmente la regulación juega un papel critico en promover la adopción de mejores prácticas de manejo forestal. Esto es ilustrado en la Figura $\mathrm{N}^{\circ} 2$, donde se observa que la claridad de las regulaciones se relaciona con las tasas de adopción. En otras palabras, las tasas de adopción fueron mayores para prácticas que fueron claramente obligadas por ley. La diferencia en las tasas de adopción entre prácticas claramente obligatorias y no obligatorias varió entre paises (la diferencia fue de $36 \%$ en Brasil, $12 \%$ en Bolivia y $18 \%$ en Perú), pero el patrón es consistente (siendo la única excepción en el Perú, donde las prácticas implicitas o recomendadas fueron más adoptadas debido a que los beneficios económicos son visibles en el corto plazo). 


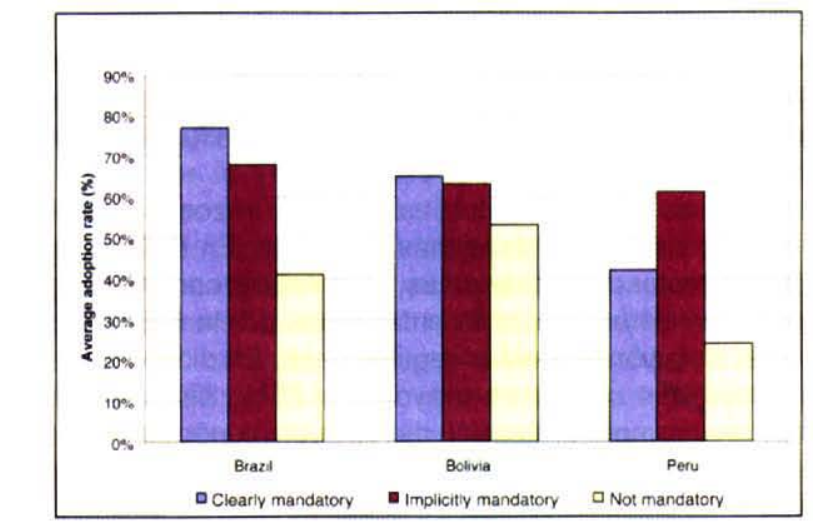

\section{Figura $\mathrm{N}^{\circ} 2$ \\ CLARIDAD DE LAS REGULACIONES Y TASAS DE ADOPCIÓN}

En Bolivia, el nivel promedio de adopción aumenta también con una mayor simplicidad (y menores costos de aplicación) de los elementos. Al aumentar los costos de inversión, las tasas promedio de adopción disminuyen de $60 \%$ a $52 \%$. En el Perú, la adopción disminuye cuando los beneficios económicos de las prácticas son a largo plazo, su aplicación requiere de altos costos de inversión y las normas no son exigentes.

En los tres países sólo una baja porción de la variabilidad en las tasas de adopción pudo ser explicada por estos factores (claridad regulatoria, plazo de los beneficios económicos, costos de inversión). En total, los valores ajustados de $\mathrm{R}^{2}$ de los análisis de regresión variaron entre 0,07 en Perú a 0,2 en Brasil. Estos valores bajos sugieren, ya sea que hay otros factores que juegan un papel o que las variables independientes no fueron medidas con suficiente precisión.

\section{Variabilidad en la Adopción de las Prácticas Entre Empresas}

Un segundo análisis se dirigió a responder la pregunta: ¿Por qué es más probable que ciertas empresas adopten una práctica más que otras y qué factores parecen estar asociados con esta probabilidad? El análisis de la variabilidad en la adopción de prácticas entre las empresas en Brasil mostró que las que estaban localizadas en las Ilamadas fronteras forestales antiguas (con más de 20 años de actividad maderera industrial) están adoptando más las buenas prácticas de manejo que sus similares de fronteras recientes ${ }^{1}$. Al parecer, esta mayor tasa de adopción en las fronteras antiguas no se debe tanto a la mayor experiencia de la empresa en el negocio forestal, sino a otras caracteristicas de estas fronteras, como: mejor acceso a información, mayor percepción de escasez del recurso forestal y mayor presión por cumplir las regulaciones. Además, se encontró que el tamaño del área aprovechada anualmente, el entrenamiento de trabajadores forestales, las inversiones realizadas, el número de trabajadores especializados y la certificación contribuyen a una mayor adopción.

\footnotetext{
' Por ejemplo, la práctica Corte direccional tuvo una tasa promedio de adopción del $42 \%$ en empresas localizadas en la frontera antigua, $13 \%$ en la frontera intermedia y $26 \%$ en la frontera reciente.
} 
Para Bolivia, las inversiones en la concesión (en particular las conducentes a la certificación) se relacionan a mayores niveles de adopción. Además, el entrenamiento periódico de los trabajadores forestales y la asistencia técnica se asocian más fuertemente con una mayor adopción de ciertas prácticas de manejo. En el caso peruano, se encontró una alta variabilidad en la adopción de las prácticas entre pequeños extractores y empresas ${ }^{2}$. Aparte de la obligatoriedad legal, otros factores que afectan la adopción son el desconocimiento de la rentabilidad del manejo, la débil administración de los recursos por el Estado (escasa supervisión y control) y la competencia desleal por madera extraida informal o ilegalmente.

\section{CONCLUSIONES Y RECOMENDACIONES}

\section{Barreras para la Adopción}

Existen varias barreras importantes a la adopción del manejo forestal empresarial en los tres paises, varias de las cuales son comunes. En el Cuadro $\mathrm{N}^{\circ} 5$ son resumidas las principales barreras encontradas de acuerdo a las encuestas realizadas y los aportes de varios grupos de actores durante los talleres de diseminación. Diversos factores externos contribuyen fuertemente a la resistencia del sector en adoptar el buen manejo.

La crisis económica de Bolivia en la época del estudio y la percepción por parte de los empresarios de la falta de seguridad física y juridica de sus concesiones forestales podria estar afectando su interés en invertir en el manejo forestal.

En Brasil, como factores externos importantes están la burocracia de los órganos reguladores, la facilidad de la extracción ilegal (competencia con empresas madereras clandestinas cuyos costos de producción son menores) y la falta de seguridad en la tenencia.

En Perú, destacan el desconocimiento de la rentabilidad del manejo por parte de los productores forestales, la débil administración de los recursos por parte del Estado (escasa supervisión y control) y la competencia desleal por madera extraída de manera informal o ilegalmente.

\section{Cuadro $\mathrm{N}^{\circ} 5$}

\section{PRINCIPALES BARRERAS A LA ADOPCIÓN DE BUENAS PRÁCTICAS DE MANEJO FORESTAL}

\begin{tabular}{|l|c|c|c|}
\hline Barreras Principales & BOL & BRA & PER \\
\hline Inseguridad juridica y fisica en tenencia de la tierra y la protección de las áreas de manejo & ++ & +++ & +++ \\
\hline Competencia desleal con empresas clandestinas / sector informal & + & +++ & +++ \\
\hline $\begin{array}{l}\text { Pocas informaciones y conocimientos en las empresas sobre lo que es manejo forestal, su renta- } \\
\text { bilidad y los costos y beneficios de las buenas prácticas }\end{array}$ & + & + & + \\
\hline $\begin{array}{l}\text { Marco legal poco claro y complejo, con procedimientos excesivamente burocráticos para hacer } \\
\text { manejo forestal (altos costos de transacción) }\end{array}$ & + & ++ & ++ \\
\hline Escasez de personal calificado. Bajo nivel entrenamiento de quienes se dedican a la actividad & + & ++ \\
\hline Capacidad acceso mercado. Dificultad introducir nuevas especies al mercado, bajos precios & + & ++ & +++ \\
\hline Falta de incentivos económico-financieros para el manejo forestal & + & ++ & +++ \\
\hline
\end{tabular}

- Refleja la situación en la época del estudio, antes de implementarse efectivamente la nueva ley forestal.

[importancia relativa: +++ muy alta; ++ alta; + medianamente alta]

${ }^{2}$ En sólo tres prácticas se detectó una tendencia a mayores tasas de adopción conforme aumenta el tamaño del productor (p.ej., el censo comercial era aplicado por $47 \%$ de las empresas, pero apenas por $28 \%$ de los pequeños extractores). 


\section{Factores que Promueven la Adopción}

Los análisis para Bolivia y Brasil permitieron destacar algunos factores que influencian positivamente la adopción de prácticas de MFS con base a las asociaciones positivas entre estas y ciertas variables independientes (Cuadro $\mathrm{N}^{\circ} 6$ ). Como factor más importante resultaron el'contenido de la ley y sus regulaciones, al igual que las formas de trabajo del ente regulador.

La adopción de las prácticas de manejo refleja los requisitos definidos en la ley y los reglamentos forestales, asi como la capacidad de hacerlos cumplir por el Gobierno.

En el caso de Bolivia, esta conclusión confirma el valor e importancia de la reforma en las políticas forestales hace 10 años, como mecanismo e incentivo para el desarrollo de este sector y el mejor uso de los recursos forestales, protegiendo su capacidad productiva hacia el futuro.

Los resultados obtenidos en este pais confirmaron las observaciones de Blate et al. (2001), quienes mencionaron la importancia de la productividad y consideraciones de costos, las leyes y sus regulaciones, la presión externa y la certificación, la asistencia técnica y un suministro oscilante de especies de alto valor.

Este estudio también apoyó algunas de las conclusiones de Putz et al. (2000) y Karsenty (2001), que señalaron como obstáculos a la adopción del MFS una insuficiente presión del ente regulador y del mercado, incentivos económicos inadecuados (ya sea proporcionados por el mercado o por los gobiernos), inestabilidad institucional, falta de capital y personal entrenado, equipo inapropiado, fluctuaciones en los mercados de madera, ignorancia y resistencia al cambio.

En Brasil, la adopción de las prácticas aparenta haber seguido un patrón debido al grado de monitoreo ejercido por el gobierno. Un diagnóstico realizado en 1995 (Silva, 1997) en la región de Paragominas (la principal región productora de madera del Estado de Pará) reveló que los planes de manejo aprobados por IBAMA no estaban siendo implementados plenamente en el campo, siendo uno de los principales problemas identificados la falla en el control gubernamental en inspeccionar y hacer cumplir esos planes.

Ese informe contribuyó para que en 1996 IBAMA iniciase inspecciones para evaluar la calidad de los planes de manejo en la Amazonía Legal. De 866 planes de manejo aprobados en 1998 sobre un área total declarada de 1.8 millones de hectáreas, para el 2000 habian apenas 389 planes aptos, cuya área total sumaba 185 mil hectáreas de bosques (Lentini et al., 2003).

\footnotetext{
Hay una mayor adopción en empresas localizadas en las fronteras antiguas de la Amazonia brasilera por la mayor escasez de materia prima y una mayor seguridad en la tenencia; además, los empresarios poseen una percepción más clara de los beneficios ambientales (menores impactos), sociales (disminución de accidentes) y económicos (reducción de costos) de las prácticas de manejo.
} 
En el caso peruano, la ley anterior, vigente en el periodo del estudio, tuvo un rol importante como factor de adopción, ya que contemplaba obligaciones de manejo forestal para los contratos por superficies mayores a 1000 ha por un plazo de 40 años renovables; de otro lado, la legislación forestal se convirtió también en un factor de no adopción debido a que se estableció una nueva modalidad de acceso al bosque, más sencilla, a través de contratos de corta duración que no incluian obligaciones en cuanto a mantener la productividad del bosque a largo plazo, lo que tuvo como resultado una extracción descontrolada y una industria forestal obsoleta (Hidalgo, 2003, Colán et al., en prensa).

\section{Cuadro $N^{\circ} 6$ \\ PRINCIPALES FACTORES QUE EJERCEN UNA INFLUENCIA POSITIVA EN LA ADOPCION DE BUENAS PRÁCTICAS DE MANEJO FORESTAL EN BOLIVIA Y BRASIL.}

\begin{tabular}{|l|c|c|}
\hline Factores de Mayor Influencia Positiva en la Adopción* & BOL & BRA \\
\hline Legislación - Obligatoriedad en las regulaciones & ++ & ++ \\
\hline Área y volumen aprovechado anualmente - En Brasil, el tamaño del área aprovechada anualmente & ++ & ++ \\
\hline $\begin{array}{l}\text { Nivel de inversiones realizadas - En Bolivia, la obtención de créditos bancarios para hacer inversiones } \\
\text { y la inversión en entrenar los trabajadores. En Brasil, inversiones en entrenamiento de los trabajado- } \\
\text { res forestales, mejoras en seguridad laboral y reforestación }\end{array}$ & ++ \\
\hline $\begin{array}{l}\text { Contratación de trabajadores permanentes / En Bolivia referido a la proporción de trabajadores per- } \\
\text { manentes; en Brasil el énfasis es en el equipo técnico) }\end{array}$ & ++ \\
\hline Número de años en la actividad forestal & ++ \\
\hline Empresa en proceso de certificación & ++ & + \\
\hline Existencia de asistencia técnica & ++ & + \\
\hline Mayorrespeto del responsable de la concesión porla opinión del forestal enla toma de decisiones demanejo & ++ & + \\
\hline Localización de la empresa, referida a la edad de la frontera forestal & + & ++ \\
\hline Porcentaje de producción exportado & + & \\
\hline
\end{tabular}

- En el caso del Perú, no se realizó el análisis sobre los factores de influencia.

[Influencia del factor en la adopción: muy positiva (++) o positiva (+); en blanco: no importante].

\section{Estrategias y Recomendaciones Principales para Aumentar la Adopción}

Basados en los resultados del análisis presentado en las secciones anteriores, en cada pais se identificó estrategias especificas y recomendaciones especificas para mejorar la adopción del MFS en escala empresarial. Entre las estrategias en común destacan:

Mayor capacitación técnica a los diferentes actores involucrados en el manejo forestal, desde gerentes, profesionales y técnicos hasta trabajadores.

En el caso de Brasil y Perú, se hace necesario revisar la normativa forestal a fin de simplificar y hacer más claras las normas existentes y describir explícitamente cuáles prácticas de manejo importantes deben ser implementadas.

Crear incentivos específicos para el manejo forestal, como: mayor acceso a información, asistencia técnica de calidad, créditos más flexibles, etc. 
Mejorar los sistemas de monitoreo y control por las propias empresas forestales.

Fortalecer la administración forestal y las instituciones de investigación y extensión forestal.

De otro lado, como parte de los talleres y reuniones de presentación de resultados preliminares del estudio en cada país se recogió algunas recomendaciones que complementan los puntos anteriores, pudiéndose destacar las siguientes:

Mejorar la seguridad jurídica de las áreas de manejo.

Difundir más ampliamente la legislación forestal y sus regulaciones.

Fortalecer la capacidad institucional de las agencias reguladoras (p.ej. personal técnico capacitado, equipamiento logístico y presencia en las áreas rurales) de manera de asegurar el control de la aplicación de las prácticas de MFS consideradas en la legislación forestal y sus regulaciones.

Promover y desarrollar al interior de las empresas la implementación de sistemas de evaluación de las respuestas del bosque a las intervenciones y de control de calidad (monitoreo) de las prácticas de manejo forestal que se adoptan en las operaciones forestales.

Fomentar el concepto de manejo "adaptativo" que incluya tanto la evaluación permanente de resultados y la efectividad de las prácticas, como la capacitación del personal y la mejora progresiva de las prácticas de manejo.

Desarrollar programas de asistencia en mercadeo y comercialización de nuevas especies e incentivos para la inversión en la industria forestal, de manera de dar mayor valor económico al bosque y generar mayores beneficios del manejo forestal para hacerlo más atractivo.

Establecer incentivos ligados a la adopción progresiva de buenas prácticas de manejo.

Generar y diseminar informaciones técnicas confiables en apoyo al manejo forestal (p.ej., resultados de investigaciones, manuales prácticos etc.).

Difundir entre los responsables del manejo forestal (en todos los niveles) los resultados de investigaciones realizadas, a fin de promover la adopción de nuevas prácticas y/o la mejora de las ya existentes que ayuden a implementar un buen manejo forestal; en particular, llevar a cabo más estudios económicos del costo/ beneficio de adoptar diferentes prácticas de manejo forestal. 
herramienta clave para asegurar la adopción de prácticas de manejo forestal de manera progresiva y segura.

Incluir en los planes de manejo la aplicación de prácticas de silvicultura postaprovechamiento para garantizar la supervivencia y el desarrollo de la regeneración natural.

Identificar y promover incentivos para el aprovechamiento sostenible de los diferentes productos no maderables y apoyar su comercialización a efectos de obtener los mayores beneficios y rentabilidad del bosque.

Mejorar la calidad de la formación profesional y técnica en el área forestal, apoyando a las carreras o cursos de especialización forestales.

Responsabilizar a los ingenieros forestales en el proceso de planificación e implementación del manejo forestal, así como el monitoreo de aplicación efectiva de las prácticas de manejo.

Aumentar el apoyo internacional para invertir en estrategias y políticas que promuevan la adopción de prácticas de MFS en bosques tropicales.

\section{RECONOCIMIENTOS}

Este estudio fue apoyado por Agencia Norteamericana para el Desarrollo Internacional (USAID/EGAT). Se agradece también en cada uno de los paises a las empresas forestales muestreadas y su personal.

\section{REFERENCIAS}

Applegate, G., F. E. Putz, L.K. Snook, 2004. Who Pays for and Who Benefits from Improved Timber Harvesting Practices in the Tropics? Lessons learned and information gaps. CIFOR, Bogor, Indonesia. $43 p$.

Barreto, P., P. Amaral, E. Vidal, C. Uhl, 1998. Costs and benefits of forest management for timber production in eastern Amazonia. Forest Ecology and Management 108: 9-26.

Blakeney, J., R. Z., Donovan, S., Higman, R. and Nussbaum, 1998. Certifier evaluation and field test of the CIFOR C\&I. CIFOR, Bogor, Indonesia. (Unpubl. Report.).

Blate, M. G., F. E., Putz, J. C. and Zweede, 2001. Progress towards RIL adoption in Brazil and Bolivia: Driving forces and implementation successes. In: International Conference on Application of Reduced Impact Logging to Advance Sustainable Forest Management: Constraints, Challenges and Opportunities. 26 Feb 1 March 2001, Kuching, Sarawak, Malaysia.

Bruijnzeel, L. A. and W. R. S.Critchley, 1994. Environmental impacts of logging moist tropical forests. International Hydrological Programme / IHP Humid Tropics Programme Series No. 7. UNESCO - IHP MAB. Paris, France. $48 \mathrm{p}$.

Camacho, 0., 2003. Directriz técnica sobre tarifa de regulación forestal para áreas con plan de manejo forestal. La Gaceta Forestal, Año 1, Número 1. Santa Cruz, Bolivia. 2 p. 
Cámara Forestal de Bolivia, 2006. Nota de Prensa CFB/27/2006, Santa Cruz, 12 de julio, 2006.

Colán, V., C., Sabogal and L. Snook, (en prensa). Diagnóstico de prácticas de manejo forestal en la Amazonia baja e implicaciones para promover el buen manejo forestal. (Monografia).

Colán, V., C. Sabogal, M. Boscolo, J. Smith L. Snook, y O. Galván, (en prensa). La extracción maderera en la Amazonia peruana: Diagnóstico de prácticas e implicaciones para promover el manejo forestal. Revista Recursos Naturales y Ambiente (CATIE).

Contreras A., y M. T. Vargas, 2001. Dimensiones Sociales, Ambientales y Económicas de las Reformas en la Politica Forestal de Bolivia. Washington DC: Forest Trends, Center for International Forestry Research.

Dykstra, D. and R. Heinrich, 1996. FAO - Model code of forest harvesting practice. Food and Agriculture Organization of the United Nations. FAO, Rome, Italy. 85 p.

FAO, 1998. Guidelines for the Management of Tropical Forests - 1. The production of wood. FAO Forestry Paper 135. Rome, Italy. 293 p.

FAO, 2001. State of the world's forests. 'Rome, Italy.

FAO, 2005. Global Forest Resources Assessment. FAO Forestry Paper 147. Rome, Italy. 348 p.

Fimbel, R., A. Grajal, J. Robinson (eds.), 2001. Conserving Wildlife in Managed Tropical Forests. Columbia University Press, New York.

Fredericksen, T.S., and F. E. Putz. 2003. Silvicultural intensification for tropical forest conservation. Biodiversity and Conservation 12:1445-1453.

Freitas, J. V. y A.C. Hummel, (en prensa). Situación del manejo forestal sostenible en la Amazonía brasileña. Revista Recursos Naturales y Ambiente (CATIE).

Galarza, E. y K. La Serna, 2005. Las concesiones forestales en el Perú: ¿Cómo hacerlas sostenibles? In: Barrantes, R., et al. (eds.). La politica forestal en la Amazonia andina. Estudios de caso: Bolivia, Ecuador y Perú. Lima, Perú, Centro de Investigación, Universidad del Pacifico.

Hammond, D. S.; P. van der Hout, R. J. Zagt, G. Marshall, J. Evans, and D.S. Cassells, 2000. Benefits, bottlenecks and uncertainties in the pantropical implementation of reduced impact logging techniques. International Forestry Review 2(1):45-53.

Hidalgo, J., 2003. Estado de la situación forestal en el Perú. In SEPIA (Seminario Permanente de Investigación Agraria). Perú: El problema agrario en debate, Sepia X, Mesa especial. Lima, Perú. 51 p.

Higman, S., S. Bass, S, N. Judd, J. Mayers and R. Nussbaum, 1999. The sustainable forestry handbook. London, UK, Earthscan.289 p.

Holmes, T. P., G.M. Blate, J. C. Zweede, R. Pereira Jr., P. Barreto, F. Boltz and R. Bauch, 2002.

Financial and ecological indicators of reduced impact logging performance in the eastern Amazon. Forest Ecology and Management 163: 93-110.

Hummel, A. C., 2000. Normas de acesso ao recurso florestal na Amazônia brasileira: O caso do manejo florestal madeireiro. Dissertação de Mestrado, Universidade do Amazonas (UA) e Instituto Nacional de Pesquisa da Amazônia (INPA). Manaus - AM, Brasil. 
INRENA, 2001. Perú Forestal en Números: Año 2000. Lima, PE, Ministerio de Agricultura. 89 p.

INRENA, 2006. Boletín Semestral Enero - Junio 2005. Documento de Trabajo. Instituto Nacional de Recursos Naturales Renovables - Intendencia Forestal y de Fauna Silvestre - Centro de Información Forestal (CIF). Lima, Perú. 40 p.

ITTO, 2006. Status of Tropical Forest Management 2005. International Tropical Timber Organization. ITTO Technical Series No. 24. Yokohama, Japan. 305 p.

Karsenty, A., 2001. Economic instruments for tropical forests: the Congo basin case. London, UK, IIEDCIFOR-CIRAD. (Monograph) iv. 98 p.

Lele, U., V.M. Viana, A. Verissimo, S. Vosti, K. Perkins and S.A. Husain,2000.

Forests in the Balance: Challenges of Conservation with Development. An Evaluation of Brazil's Forest Development and World Bank Assistance. World Bank, Brasilia.

Lentini, M., A. Veríssimo, e L. Sobral, 2003. Fatos florestais da Amazônia 2003. Imazon. Belém, Brasil. $110 \mathrm{p}$.

Lentini, M., D. Pereira, D. Celentano, e R. Pereira, 2005. Fatos florestais da Amazônia 2005. Belém: Imazon. $4 \mathrm{p}$.

Løyche Wilkie, M., 2001. Status and Trends in Forest Management Worldwide. 1980-2000. Forest Management Working Papers, Working Paper 6. Forest Resources Development Service, Forest Resources Division. FAO, Rome. November 2001. http://www.fao.org/DOCREP/004/Y2701E/y2701e00.htm\#Contents

Pacheco, P., 2003. Municipalidades y participación local en la gestión forestal en Bolivia en Farroukhi, Lyes (ed.), La Gestión Forestal Municipal en América Latina, CIFOR/CIID. 236 p. (disponible en www.IDRC.ca). Pearce, D., F. E. Putz and J. K. Vanclay, 2001. Sustainable forestry in the tropics: panacea or folly? Forest Ecology and Management 5839: 1-19.

Pokorny, B., C. Sabogal, J. N. M. Silva, P. Bernardo, J. Souza and J. Zweede. 2005. Compliance with reduced-impact harvesting guidelines by timber enterprises in terra firme forests of the Brazilian Amazon. International Forestry Review 7(1):9-20

Poore, D., P. Burgess, J. Palmer, S. Rietbergen and T. Synnott, 1989. No timber without trees: Sustainability in the tropical forest. A Study for ITTO. London, UK, Earthscan Publications.

Putz, F. E., D. P. Dykstra and R. Heinnch, 2000. Why poor logging practices persist in the tropics? Conservation Biology 14: 951-956.

Putz, F. E., K. H. Redford, J. G. Robinson, R. Fimbel and G. M. Blate, 2002. Biodiversity Conservation in the Context of Tropical Forest Management. The World Bank, Environment Department Papers, Paper No. 75. Washington DC, USA. 80 p.

Rice, R. E., C. A. Sugal, S. M. Ratay and G. A. B. da Fonseca,2001. Sustainable forest management: A review of conventional wisdom. Washington DC, US, Center for Applied Biodiversity Science / Conservation International. Advances in Biodiversity Science No. 3. 28 p.

Richards, M., 2000. Can sustainable tropical forestry be made profitable? The potential and limitations of innovative incentive mechanisms. World Development 28(6):1001-1016. 
Sabogal, C., J. N. M. Silva, J. Zweede, R. P. Júnior, P. Barreto e C. A. Guerreiro, 2000. Diretrizes técnicas para a exploraçāo de impacto reduzido em operaçōes florestais de terra firme na Amazônia brasileira. Embrapa Amazônia Oriental, Documentos 64. 52 p.

Sabogal, C., M. Lentini, B. Pokorny, P. Bernardo, F. Massih, L. Sobral, J.N.M. Silva, J. Zweede, M. Boscolo e A. Verissimo, 2005. Manejo Florestal Empresarial na Amazônia Brasileira: Restrições e Oportunidades para a Adoção de Boas Práticas de Manejo. Relatório Final. Belém: Cifor-Imazon-EmbrapaFFT. 107 p. Disponivel nos sites: http://www. cifor.cgiar.org/brazil e www.mma.gov.br/pnf.

Sabogal, C., M. Lentini, B. Pokorny, J. N. M. Silva, J. Zweede, A. Veríssimo e M. Boscolo, 2006. Manejo Florestal Empresarial na Amazônia Brasileira: Restriçōes e Oportunidades - Relatório Sintese. CIFOR Embrapa - Imazon - IFT. Belém - Pará, Brasil. 71 p.

Sabogal, C., M. Lentini, B. Pokorny, J. N. M. Silva, J. Zweede, A. Verissimo y M. Boscolo, (en prensa). Manejo forestal empresarial en la Amazonia brasileña: Restricciones y oportunidades para la adopción de buenas prácticas de manejo. . Revista Recursos Naturales y Ambiente (CATIE).

Silva, J. N. M., 1997. Avaliação de planos de manejo florestal sustentável na região de Paragominas, Pará. In: Seminário sobre o Diagnóstico dos Projetos de Manejo Florestal no Estado do Pará - Fase Paragominas, 1996, Paragominas, PA. Anais. Belém - Embrapa Amazônia Oriental, Documentos, 106. 1997. 133 p.

Smith, J., V. Colán, C. Sabogal y O. Galván, 2003. De la extracción migratoria hacia la intensificación: Implicaciones para un mejor manejo forestal en la Amazonia peruana. Bosques Amazónicos (Perú), 33: 1-13.

Smith, J., V. Colan, C. Sabogal and L. Snook, 2006. Why policy reforms fail to improve logging practices: The role of governance and norms in Peru. Forest Policy and Economics 8: 458-469.

Snook, L., L. Quevedo, M. Boscolo, C. Sabogal y R. Medina, (en prensa). Avances y limitantes en la adopción del manejo forestal sostenible en Bolivia. Revista Recursos Naturales y Ambiente (CATIE).

Superintendencia Forestal, 2004. Informe anual 2003. Imprenta Sirena, Santa Cruz., Bolivia.

Verissimo, A., E. Lima e M. Lentini, 2002. Pólos Madeireiros do Estado do Pará. Belém: Imazon. 75 p. 